\title{
Neurocutaneous melanosis
}

\author{
C. G. HARPER AND D. G. T. THOMAS \\ From the Institute of Neurological Sciences, Southern General Hospital, Glasgow
}

SYNOPSIS A case of neurocutaneous melanosis in a young child is described. The initial clinical and neuroradiological diagnosis was aqueduct stenosis.

Malignant melanoma of the leptomeninges is occasionally associated with giant benign hairy naevi. This syndrome is known as neurocutaneous melanosis. Such a case is presented here because the syndrome does not seem to be widely known.

\section{CASE REPORT}

G.W., a 14 month old boy, presented with a history of vomiting two or three times daily for six weeks and of one generalized convulsion three weeks earlier. His birth history and development were normal and there was no family history of neurological disease.

On admission he was conscious but irritable. His head circumference was in excess of 2 SD above the mean for his age. Transillumination confirmed the presence of hydrocephalus. The anterior fontanelle was bulging but he had no neck stiffness. The fundi appeared normal. Cranial nerve function was normal and no neurological deficit was found in the limbs. The plantar responses were flexor. Numerous hairy naevi were present over the scalp, trunk, and limbs. No other abnormality was found on general examination.

The haemoglobin was $12 \cdot 3 \mathrm{~g} / 100 \mathrm{ml}$ and the white blood count $13,200 / \mathrm{mm}^{3}$. Urea and electrolytes were normal. Lumbar puncture produced clear cerebrospinal fluid (CSF) with a protein concentration of $740 \mathrm{mg} / 100 \mathrm{ml}$ and a normal sugar content. There was no increase in cells and the CSF was sterile. Electroencephalography (EEG) showed evidence of a diffuse structural disorder, more marked over the right hemisphere than the left. Plain skull $x$-ray films showed the changes of raised intracranial pressure and hydrocephalus.

The right lateral ventricle was catheterized under neuroleptanalgesia. The CSF pressure was moderately raised. Conray ventriculography showed symmetrical dilatation of the lateral ventricles and a widened third ventricle. Only a trickle of contrast medium flowed through the aqueduct into the fourth ventricle, which did not appear to be enlarged or displaced. A probable diagnosis of aqueduct stenosis was made and a ventriculoperitoneal shunt was inserted under general anaesthesia the same day. The child appeared well for a few hours postopera

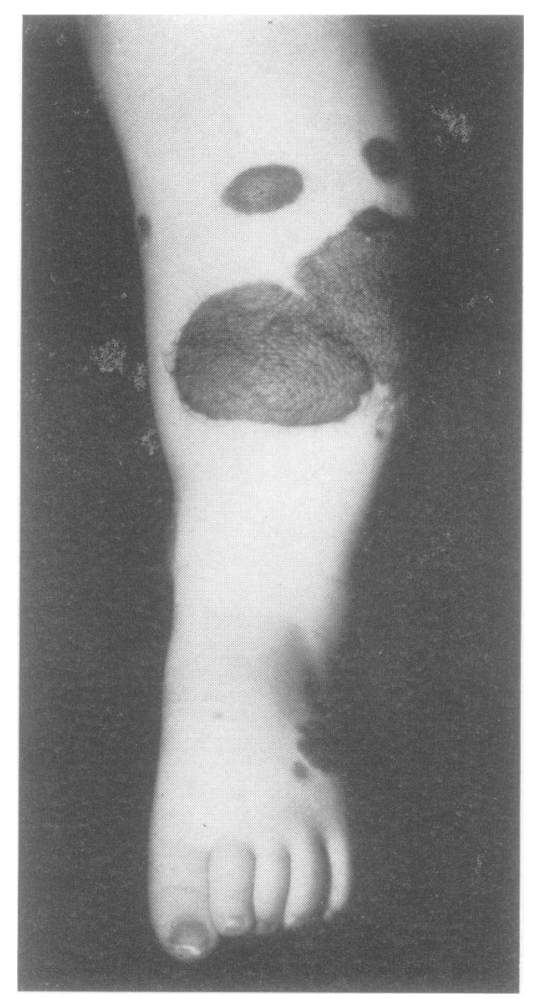

FIG. 1. Benign giant hairy naevi on the leg. 


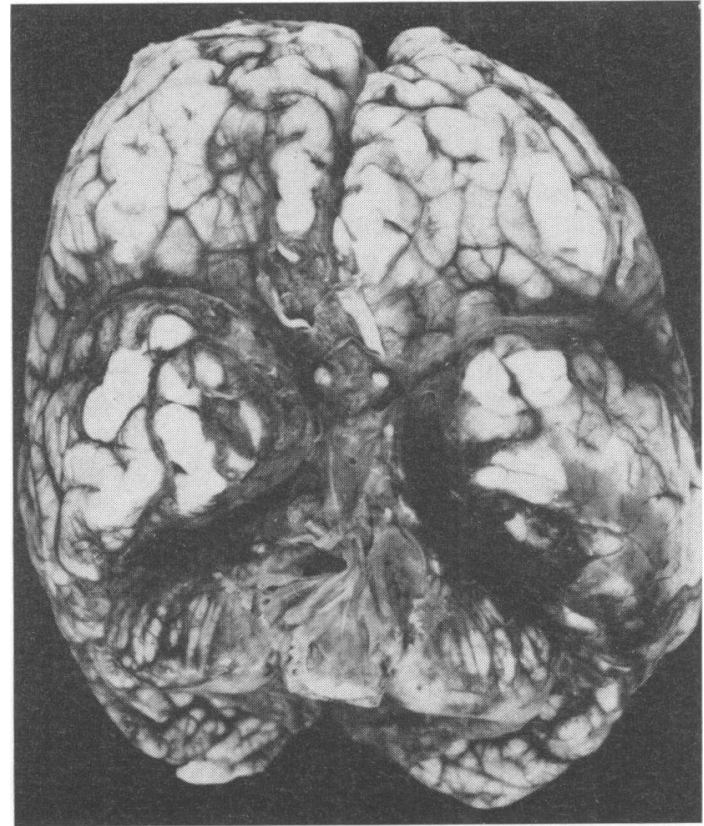

FIG. 2. Dark thickened opaque meninges around the base of the brain. tively but then suddenly became apnoeic. He died in spite of endotracheal intubation and ventilation.

NECROPSY FINDINGS There were many hairy pigmented moles over all parts of the body, most numerous on the legs and back, the largest being $6 \mathrm{~cm}$ in diameter (Fig. 1). Several were hairless but none showed any of the macroscopic features associated with malignant change such as crusting, bleeding, or satellitism. Histological examination of random blocks taken from several moles showed typical benign naevi. Other abnormalities were confirmed to the central nervous system.

Neuropathology The cranial suture lines were poorly fused for a child of 14 months and the bones of the calvaria were thin.

External examination of the brain showed grey, thickened, and opaque meninges, particularly in the hypothalamic region (Fig. 2) and on the ventral surface of the brain-stem. The roof of the fourth ventricle was ballooned and composed of a thickened membrane of grey tumour tissue.

In $1 \mathrm{~cm}$ coronal slices of the fixed brain there was marked dilatation of both lateral ventricles and of the third ventricle. The ependyma was normal. Dark grey tumour tissue was most conspicuous at the base of the brain and within each Sylvian fissure. At the medial end of each Sylvian fissure tumour was infiltrating into the adjacent cortex (Fig. 3).

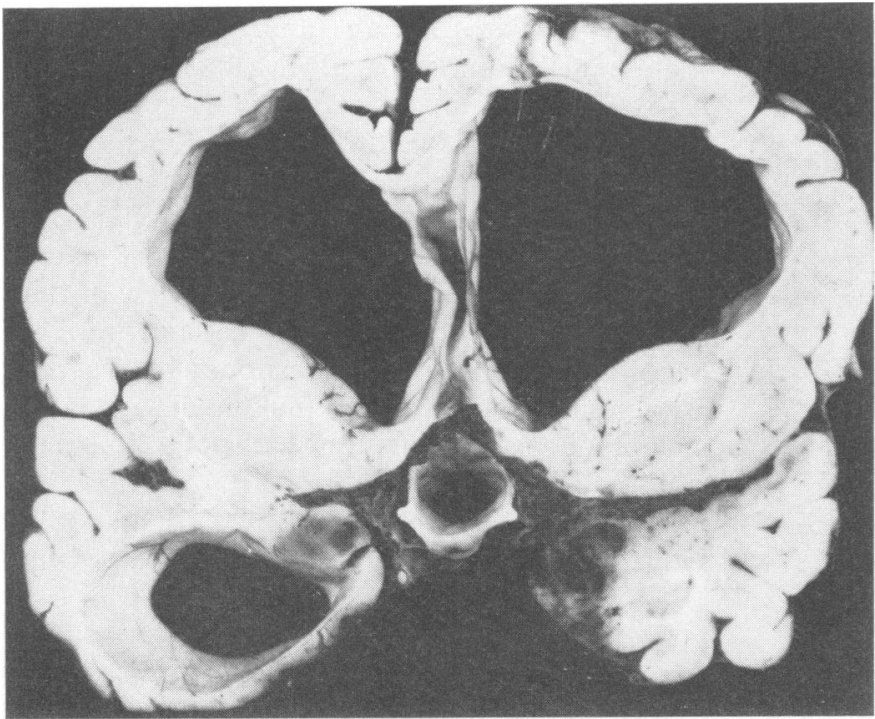

FIG. 3. Tumour tissue within each Sylvian fissure is infiltrating into the adjacent cortex. There is marked hydrocephalus. 


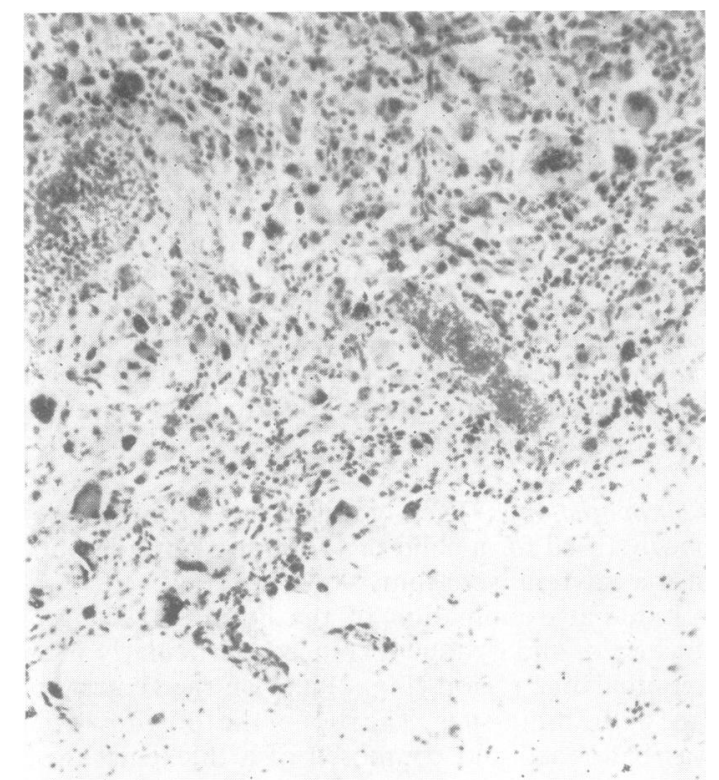

FIG. 4. Typical malignant melanoma with marked pleomorphism and bizarre giant cells in the subarachnoid space. $H$ and $E, \times 250$.

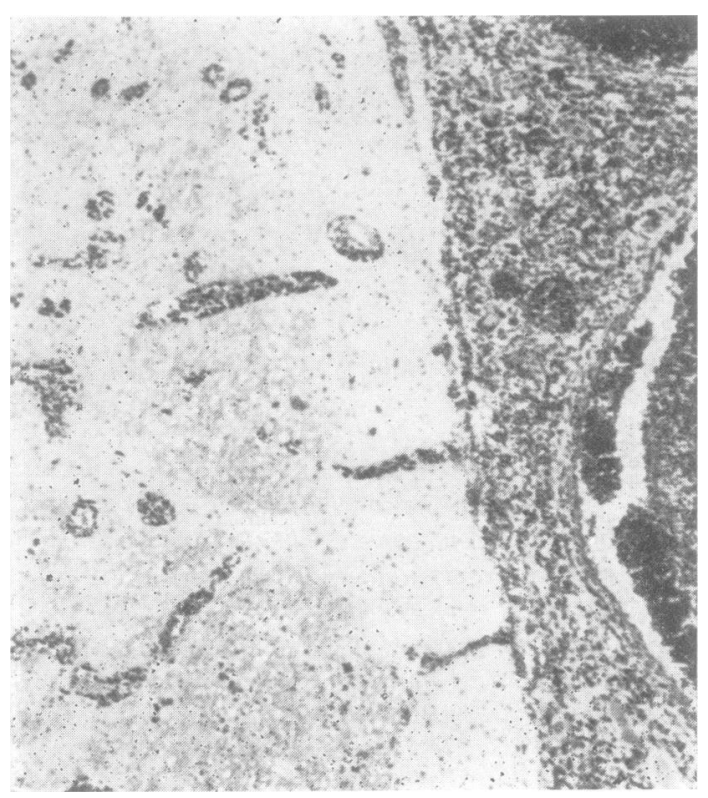

FIG. 5. Distinctive perivascular infiltration of the cortex by malignant melanoma cells. $H$ and $E, \times 100$.
The fourth ventricle was so grossly enlarged that there was severe deformity of both cerebellar hemispheres with flattening of their inferior surfaces and upward displacement of the superior cerebellar vermis to such an extent that a reversed tentorial hernia was present.

The spinal cord was ensheathed by tumour tissue which in places was up to $0.5 \mathrm{~cm}$ thick.

Histology In all specimens of brain and spinal cord the subarachnoid space was diffusely infiltrated by tumour. The features were typical of malignant melanoma. There were numerous mitoses and marked pleomorphism with bizarre giant cells was present (Fig. 4). Melanin pigment was prominent. Where invasion of neural tissue had occurred there was a distinctive perivascular arrangement of the melanoma cells (Fig. 5).

\section{DISCUSSION}

Neurocutaneous melanosis has been well reviewed in the literature (Hoffman and Freemanto

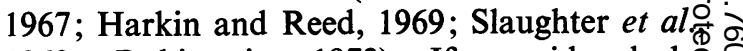
1969; Rubinstein, 1972). If considered bP clinicians an early diagnosis can sometimes be established by cytological study of the CSF, but on two occasions in the present case the ce $\mathbb{B}$ count in lumbar CSF was within normal limits

In this case the correct diagnosis was nơ entertained. Neuroradiological investigation $\overrightarrow{\mathrm{s}}$ suggested that the hydrocephalus was due to aqueduct stenosis and consequently further diagnoses were not sought.

The problem of an unidentified primary malignant melanoma is always raised in such a case. However, in the absence of an obvious primary tumour, even in less common sites such as the retinae or the nasopharynx, or any visceral metastases an occult primary tumour is unlikely. In the present case not every naevus was examined histologically but, in view of their benign macroscopic appearance and random histological sampling, these should be regarded as benign. Furthermore, malignant cutaneous melanoma is extremely unusual in young children (Willis, 1967).

We hope that this case report will make the syndrome more widely known.

\section{REFERENCES}

Harkin, I. C. and Reed, R. J. (1969). Tumors of the peripheral nervous system. Atlas of Tumor Pathology, 2nd 
series, fascicle 3, pp. 99-106. Armed Forces Institute of Pathology: Washington.

Hoffman, H. J., and Freeman, A. (1967). Primary malignant leptomeningeal melanoma in association with giant hairy naevi. Report of two cases. Journal of Neurosurgery, 26 , 62-71.

Rubinstein, L. J. (1972). Tumors of the central nervous system. Atlas of Tumor Pathology, 2nd series, fascicle 6, pp. 309-311. Armed Forces Institute of Pathology: Washington.

Slaughter, J. C., Hardman, J. M., Kempe, L. G., and Earle, K. M. (1969). Neurocutaneous melanosis and leptomeningeal melanomatosis in children. Archives of Pathology, 88, 298-304.

Willis, R. A. (1967). The melanomata. In Pathology of Tumours, pp. 915-936, 4th edn. Butterworths: London. 